\title{
Issues in strength models for unidirectional fibre-reinforced composites related to Weibull distributions, fibre packings and boundary effects
}

\author{
Yentl Swolfs* ${ }^{* 1}$, Ignaas Verpoest ${ }^{1}$, Larissa Gorbatikh ${ }^{1}$ \\ ${ }^{1}$ Department of Materials Engineering, KU Leuven, Kasteelpark Arenberg 44 bus 2450, \\ Belgium \\ *Corresponding author: Y. Swolfs (yentl.swolfs@mtm.kuleuven.be)
}

\begin{abstract}
Three issues are investigated that may influence the accuracy of strength models for unidirectional composites. Firstly, most authors limit themselves to 25-100 single fibre tests to determine the Weibull distribution. This is insufficient to accurately determine this crucial input parameter, leading to significant errors in the predicted composite failure strains. Secondly, random, square and hexagonal fibre packings are shown to lead to a similar predicted failure strain and cluster development. This is the first strong indication that the type of fibre packing has little influence on modelling predictions. Finally, boundary fibres introduced at the model perimeter were shown to prevent preferential cluster formation near the model perimeter. This makes the models less sensitive to the number of fibres. Boundary fibres do not influence the early cluster development, but do increase the critical cluster size. The results provide guidelines for when such boundary fibres should be included.
\end{abstract}

Keywords: A: Polymer-matrix composites (PMCs); B: Strength; C: Modelling; C: Stress concentrations; Unidirectional composites.

\section{Introduction}

Carbon fibre composites combine low density with high stiffness and strength, making them a suitable material for lightweight applications. Currently, composite structures are overdesigned due to a lack of reliable predictive models for their mechanical properties. The World Wide Failure Exercise attempted to predict failure of multidirectional composites under triaxial loading conditions [1]. These predictions are extremely challenging due to the inherent complexity of the problem as well as the difficult experimental validation $[1,2]$.

Under uniaxial loading conditions, the longitudinal tensile failure and damage development of unidirectional (UD) composites are better understood in the composites community. Upon increasing the applied strain, more and more fibres start failing according to the Weibull distribution of their strength. Each broken fibre locally stops carrying load and sheds that load to nearby fibres. The matrix surrounding the fibre break is loaded in shear and transfers stress back onto the broken fibre. The shear stress transfer in the matrix also causes nearby fibres to experience stress concentrations over a certain length [3-9], increasing their failure probability. Eventually, these stress concentrations lead to the development of clusters of fibre breaks [10-14], which further intensify the stress concentrations. One of these clusters will reach a certain critical size, causing unstable propagation and hence final composite failure. Despite the relative simplicity of this failure process compared the World Wide Failure Exercise, issues arise at various points of modelling this failure process. The two main issues are (1) the Weibull distribution for fibre strength and (2) the stress distribution around fibre breaks. 
The first issue is selecting an appropriate type of Weibull distribution. The standard Weibull probability distribution $P$ for fibre strength is:

$$
P=1-\exp \left(-\left(\frac{L}{L_{0}}\right)\left(\frac{\sigma_{f}}{\sigma_{0}}\right)^{m}\right),
$$

with $L$ the characteristic gauge length, $L_{0}$ the reference gauge length, $\sigma_{f}$ the fibre strength, $\sigma_{0}$ the scale parameter and $m$ the shape parameter or Weibull modulus [15]. The parameters $\sigma_{0}$ and $m$ are typically calculated from single fibre tests at gauge lengths $L_{0}$ between 10 and $50 \mathrm{~mm}$. Most strength models, however, require the Weibull distribution at gauge lengths $L$ in the order of micrometers [16-18]. Several authors have mentioned that equation 1 leads to overestimations of fibre strength at short gauge lengths [19-21].

In the experimental determination of single fibre strengths the following difficulties have been reported. Thomason [22] mentions that a minimum strength is needed to extract a fibre from a yarn prior to testing single fibres. This exclusion of the weakest fibres results in a deviation from linearity at low strength in Weibull plots. Such deviations are common [22-24], although some authors were able to avoid them [25]. Thomason also illustrates that a low number of tests may falsely lead to the conclusion of more than one flaw population. The number of tests to extract Weibull parameters typically ranges between 20 and $50[23,24]$, while only in a few studies sample sizes of 100 or more were used [5,26]. Issues with the number of required tests were reported by Berger and Jeulin [27]. They proved that 30 tests are insufficient to accurately determine Weibull shape and scale parameters. Unfortunately, Berger and Jeulin did not determine the magnitude of variations this introduces in strength predictions.

A second issue is the stress redistribution in the composite when fibres break. The simplest approximation is an undamaged, linear elastic matrix, linear elastic fibres and an intact fibre-matrix interface. This leads to infinite stress concentrations around the fibre breaks, but in reality they are released through three possible mechanisms or a combination thereof. Matrix shear yielding is typical in thermoplastic composites, but also locally occurs in thermoset versions. Fibre-matrix debonding is observed in composites with poor adhesion, while matrix cracks are typical in composites with good adhesion and a brittle matrix [28]. The mechanisms that occur can have a strong influence on the strength predictions, as shown extensively in literature [9,12,16,29-32].

Most strength models use either square [33,34] or hexagonal packings [35,36], while in reality fibres are randomly arranged [37]. Random fibre packings introduce variations in the stress concentrations [3,38,39], while they are deterministic for square and hexagonal packings. If an intact fibre almost touches a broken fibre, then the stress concentration is about twice as high as in hexagonal packings [3]. This is the case even though the overall fibre volume fraction is the same. Moreover, the stress recovery length in broken fibres in random packings is known to be shorter [3]. Since both effects counteract each other, the influence of packing type on the composite strength remains difficult to predict.

This paper investigates three possible issues in strength models for UD composites: Weibull distribution, fibre packing and boundary effects. These three issues have sometimes been taken for granted in literature, without a proper assessment of their influence on model predictions. 


\section{Model description}

\subsection{General approach}

The model has already been extensively described in $[32,40]$, so only the main points are highlighted here. Fig. 1 presents the general approach of the strength model in a flow chart. The model starts off by creating a representative volume element (RVE) based on the input parameters described in "2.2 Input parameters". This RVE is sufficiently large to be representative for larger structures. It consists of a bundle of parallel fibres, which are split up into fibre elements. A Weibull strength is applied to each of these fibre elements prior to each simulation. The global strain $\varepsilon$ is then gradually incremented. Next, the element stress $\sigma_{i}$ is calculated as $\sigma_{i}=\varepsilon \cdot E_{f} \cdot S C F_{i}$, where $E_{f}$ is the fibre tensile modulus and $S C F_{i}$ is the stress concentration factor applied to that element. The calculation of this $S C F_{i}$ will be explained later. The strain incrementing and updating of element stresses is repeated until the first element fails.

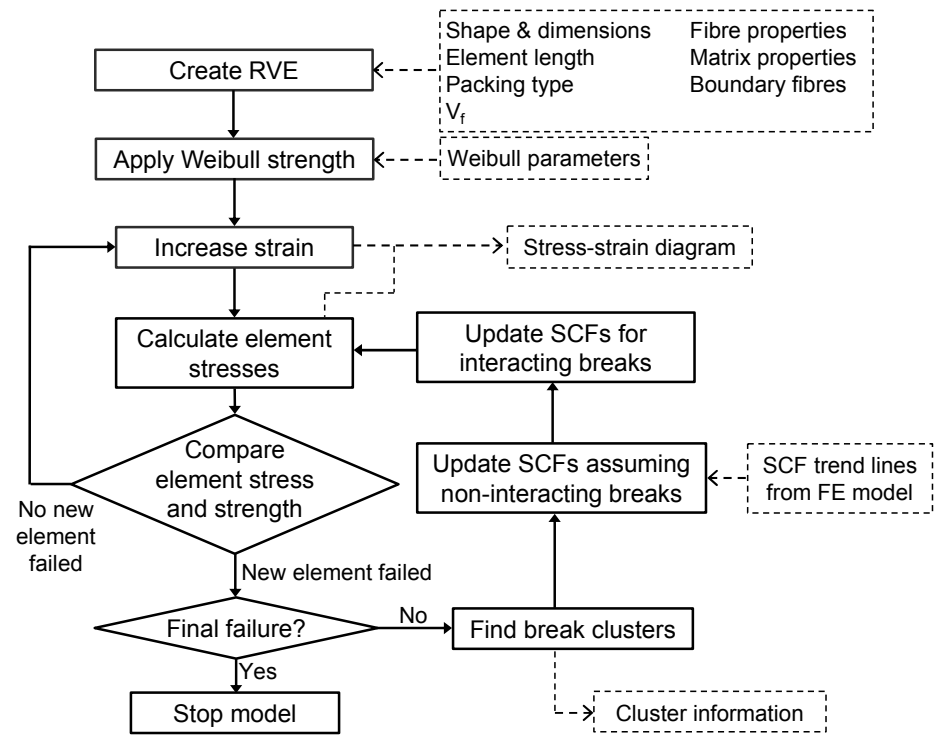

Figure 1: Flow chart of the strength model. The dashed rectangles indicate inputs and outputs.

When new fibre elements do fail, the composite failure criterion is checked. This is based on $10 \%$ of all the fibres in the RVE being broken within any axial segment with a length of 10 fibre radiuses. This somewhat arbitrary criterion was chosen as its satisfaction always coincided with an unstable propagation of fibre breaks. If the failure criterion is not satisfied, then clusters of fibre breaks are detected. Two fibre breaks are part of the same break-cluster if: (1) the surface-to-surface distance between the fibres is smaller than 2 fibre radiuses, and (2) the axial distance is less than 10 fibre radiuses. This definition corresponds to regions with significant SCFs, meaning that fibre breaks further away are primarily caused by random strength variations instead of stress concentrations. Clusters are referred to as "i-plets", where "i" is the number of fibre breaks in the cluster. The critical cluster size is defined as the number of fibres in the largest cluster in the last strain increment prior to final failure.

The SCFs are then updated by first assuming that the fibre breaks do not interact with each other. The stress redistributions were obtained from finite element (FE) solutions around a single fibre break surrounded by a matrix crack. This procedure has been described in $[3,39]$, but was modified in one aspect. A matrix crack was added around the single broken fibre, as a way to release the infinite stress concentrations at the 
perimeter of the fibre breaks. The matrix crack was stopped by the surrounding fibres, but its exact size and shape does not significantly influence the stress redistribution[32].

The SCFs are then adapted to account for possible interactions between fibre breaks by using the enhanced superposition principle [32]. This principle is based on linear superposition of the single fibre break solutions and has been previously described in Swolfs et al. [32]. It was found to be accurate in the presence of matrix cracks, as is the case here. This was true for co-planar clusters up to 5-plets, but this validation was not attempted for even larger clusters.

After applying the SCFs, the element stresses are updated again. The procedure in Fig. 1 is repeated until the failure criterion is satisfied. The model is then stopped and the output data are extracted. The composite stress is derived from the average fibre and matrix stresses. The average fibre stress is calculated by averaging stresses over all elements. The average matrix stress is calculated as the global strain multiplied by the matrix modulus.

A total of 50 strength simulations were performed for each configuration. Figures 3, 6-9 present the average value of these simulations as well as the standard deviation.

\subsection{Input parameters}

The RVE of unidirectional fibres has a cylindrical shape with a $10 \mathrm{~mm}$ length. The RVE cross-section consists of 2000 fibres in a random fibre packing with a 50\% fibre volume fraction. Each fibre is split up into 2857 elements, corresponding to a $3.5 \mu \mathrm{m}$ element length, which is also the carbon fibre radius. Tensile moduli of carbon fibre and epoxy matrix are $230 \mathrm{GPa}$ and $3 \mathrm{GPa}$, respectively. While other engineering constants of fibre and matrix affect the FE solution for stress re-distribution around a fibre break $[3,39]$, the strength model only considers axial fibre stresses. All materials are assumed to be linear elastic to simplify the analysis. Including non-linear behaviour would change the reported values, but would not change their interpretation.

The Weibull distribution is described in equation 1. The purpose here is to illustrate possible issues in strength models and not to compare model predictions to experimental data. Therefore, a Weibull distribution is chosen with a realistic Weibull modulus, and which leads to a reasonable failure strain prediction. The chosen parameters are $\sigma_{0}=3500 \mathrm{MPa}, \mathrm{m}=6$, and $L_{0}=10 \mathrm{~mm}$.

Since broken fibres near the model perimeter have fewer nearest neighbours, the SCFs on those neighbours are higher. This increases the probability for developing breakclusters at the model perimeter. To mitigate this issue, a ring of boundary fibres is added along the model perimeter. This ring is about 2 fibres thick, leading to about 270 boundary fibres in addition to the other 2000 fibres. These boundary fibres will be used throughout this study. They can carry SCFs, but cannot break and are not included in calculations of the composite stress. Their influence on the model predictions will be analysed in "3.3 Boundary effects".

\section{Results and discussion}

\subsection{Weibull distribution}

To exactly measure the Weibull distribution, an infinite number of single fibre tests is required. Nevertheless, most authors limit the number of tests to between 25 and 100 due to the cumbersome sample preparation and testing. This small number of tests 
introduces statistical errors, and may have a large influence on the predicted failure strains.

To analyse this influence, the default data set with $\sigma_{0}=3500 M P a$ and $m=6$ is considered to be the real data set. Single fibre tests are simulated by randomly generating strength values from the real data set at a $10 \mathrm{~mm}$ gauge length. This is performed for 10 sets of 25, 100, 400 and 1600 fibres. For each of these 40 data sets, the maximum likelihood approach is used to calculate the Weibull distribution. The resulting Weibull parameters are then used as input parameters in the strength model. The extrapolation of a $10 \mathrm{~mm}$ gauge length to a $0.0035 \mathrm{~mm}$ element length is assumed to be accurate, even though the accuracy of this extrapolation is still being debated in literature [19-21].

Testing only 25 fibres can lead to large errors in the Weibull parameters (see Fig. 2). Even for 400 tested fibres, the estimated Weibull parameters can still significantly deviate. The errors are especially large for the Weibull modulus $m$. This parameter is difficult to determine accurately, as it is a power law exponent in the Weibull equation. For 1600 tested fibres, the estimated Weibull parameters are reasonably accurate.

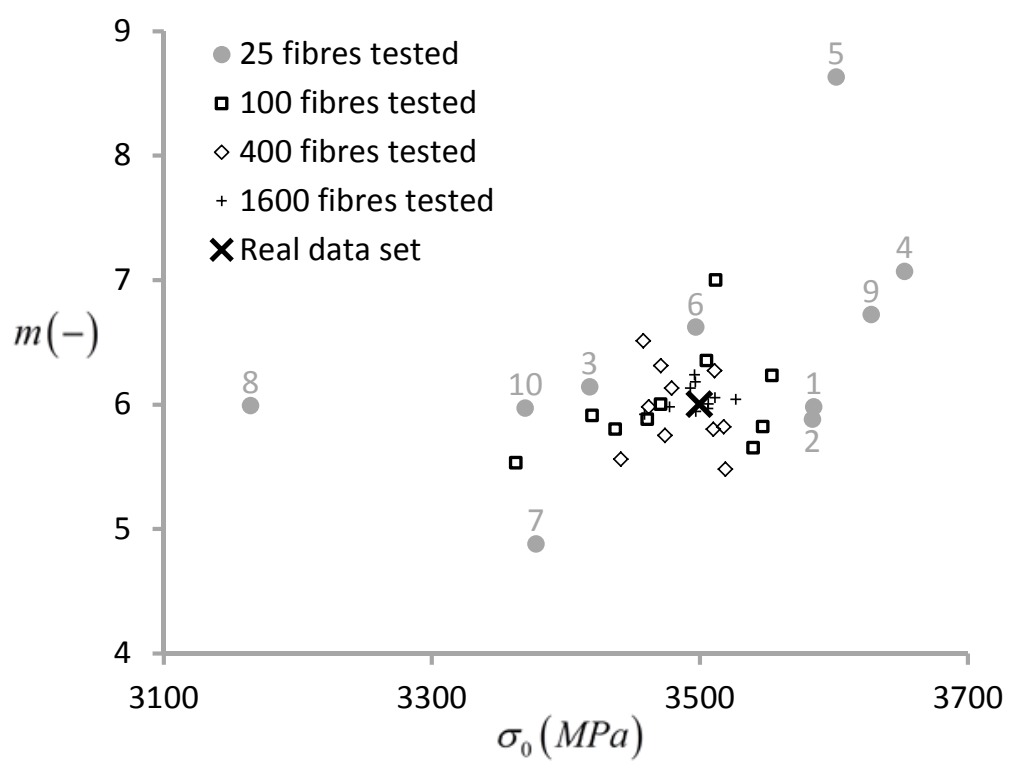

Figure 2: Summary of all Weibull parameters for 25, 100, 400 and 1600 fibres tested. The number labels for " 25 fibres tested" are added to facilitate comparison with Figure 3.

These 40 data sets are used as input in the strength model to calculate composite failure strain. Fig. 3 compares the predicted failure strains for these data sets to the predicted failure strain for the real data set. For 25 fibres tested, the predicted failure strains range between $1.70 \%$ and $2.19 \%$. This is a variation of about $25 \%$ in failure strain. One way to reduce this scatter is to test more fibres. With 100 tested fibres, the predicted failure strain ranges between $1.83 \%$ and $2.09 \%$, or a total variation of $13 \%$. This total variation reduces to $12 \%$ for 400 tested fibres and $3 \%$ for 1600 tested fibres. 


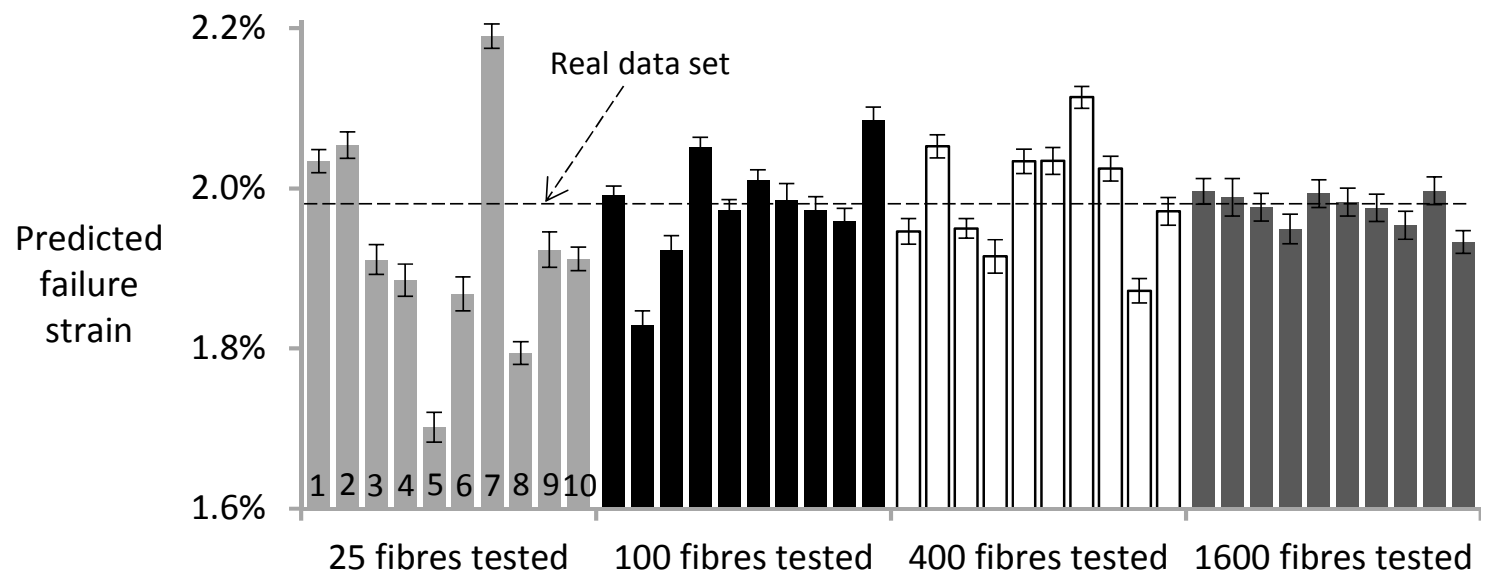

Figure 3: Predicted failure strains for 25, 100, 400 and 1600 fibres tested. The dashed line indicates the predicted failure strain for the real data set.

These results limit the predictive capabilities of strength models for three reasons. Firstly, most authors limit themselves to testing 25-100 fibres, while Fig. 3 illustrates this leads to significant errors in the failure strain predictions. Secondly, the approach used here only takes into account statistical errors. This assumes fibre strength can be measured accurately. Experimental difficulties in single fibre tests introduce even larger errors in the Weibull distribution and hence in the predicted failure strains. Thirdly, experimental errors may be skewed towards one side. Clamping effects in single fibre testing are known to be more severe for strong fibres [41], while weak fibres may break during sample preparation [22].

Instead of testing all fibres at the same gauge length, it is also possible to test them at two or more gauge lengths. It is often believed that this results in more reliable estimates of the Weibull parameters. Therefore, it is worthwhile to investigate whether the accuracy of our estimates for $\sigma_{0}$ and $m$ depends on the gauge length. We therefore simulated 100 single fibre tests at a gauge length of $10 \mathrm{~mm}$ and determined the $\sigma_{0}$ and $m$-value for this set of tests. We repeated this 5 million times, yielding 5 million estimates for $\sigma_{0}$ and $\mathrm{m}$. The same exercise was performed for gauge lengths of $25 \mathrm{~mm}$ and $100 \mathrm{~mm}$. To compare the outcomes, the $\sigma_{0}$-values for gauge lengths of $25 \mathrm{~mm}$ and $100 \mathrm{~mm}$ were rescaled to $10 \mathrm{~mm}$ using equation 1 . This rescaling used the Weibull modulus that was estimated based on the simulated 100 tests, as the real Weibull modulus will not be known in practice. The $\sigma_{0}$ results after rescaling with the estimated Weibull moduli reveal a larger spread for a gauge length of $100 \mathrm{~mm}$ (see Fig. 4a). The probability density function for the Weibull modulus however remains the same for all tested gauge lengths. This means that the strength scatter is independent of the tested gauge length. This is inherent to the Weibull distribution, as the parameter $m$ in equation 1 is independent of $L$.

The question arises why testing at a gauge length of $100 \mathrm{~mm}$ would be less accurate. The answer is revealed when the extrapolation of $\sigma_{0}$ is performed using the real instead of the Weibull modulus that was estimated based on the 100 simulated tests. Although not shown here, the probability density functions for $\sigma_{0}$ would all coincide. This means that the higher $\sigma_{0}$ scatter for a gauge length of $100 \mathrm{~mm}$ is solely due to the scatter on the estimated Weibull modulus (see Fig. $4 \mathrm{~b}$ ) that is used to extrapolate to $L_{0}=10 \mathrm{~mm}$. 
This has two crucial implications. Firstly, it implies that the gauge length of a single fibre test does not influence the accuracy of the estimates for $\sigma_{0}$ and $\mathrm{m}$. Instead, the best solution is to test the fibres at the gauge length at which the Weibull distribution is required. Secondly, it also implies that testing at multiple gauge lengths is less accurate from a statistical point of view, as this always implies extrapolations of a part of the results.

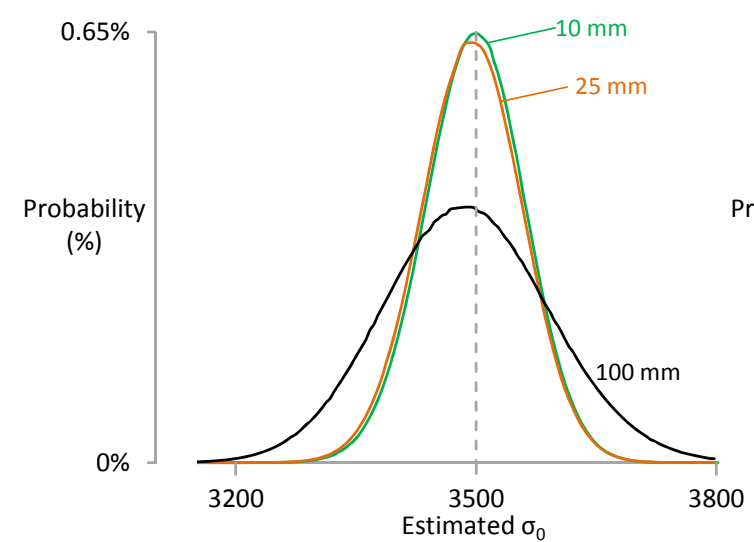

(a)

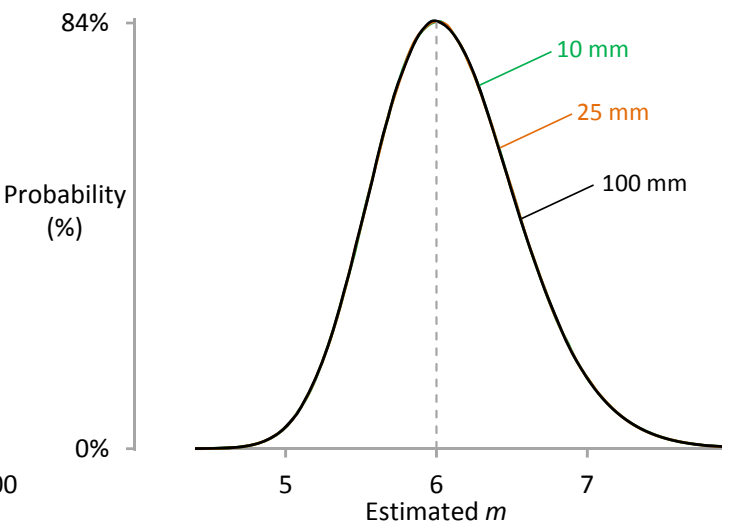

(b)

Figure 4: Measuring the Weibull parameters is more accurate if performed at the reference gauge length: (a) Weibull scale parameter $\sigma_{0}$ and (b) Weibull modulus $\mathrm{m}$. The reference gauge length $\mathrm{L}_{0}$ was assumed to be $10 \mathrm{~mm}$ and extrapolations of $\sigma_{0}$ down to this length were performed using the estimated Weibull modulus.

The results illustrate the importance of testing a sufficient number of fibres to determine the Weibull distribution. Results will converge to the real value, but this may require a number of tests that is unfeasible from a practical point of view. The development of improved testing methodologies is highly recommended to allow more data collection in a shorter time period. Measuring the Weibull distribution at gauge lengths below 10 $\mathrm{mm}$ may be even more difficult due to additional experimental errors. Unfortunately, it is exactly these gauge lengths that are relevant for most strength models. Extrapolation down to small gauge lengths causes an even larger uncertainty if the Weibull modulus is not known accurately. Unfortunately, it is especially the determination of the Weibull modulus that is troublesome (see Fig. 2). Furthermore, such short fibres may not behave according to a Weibull distribution anymore [8].

\subsection{Fibre packings}

Square and hexagonal fibre packings are commonly used, as their regular structure simplifies many existing strength models. Regular packings are clearly an assumption, as fibres are randomly packed in reality. Fig. 5 illustrates the influence of the packing on the stress redistribution in a broken fibre and intact surrounding fibres.

Fig. 5a displays some small differences for the ineffective length. The stress in the broken fibre is recovered slightly faster for random fibre packings, but regular packings lie within the standard deviations. Random fibre packings allow fibres to be closer to the broken fibres. This increases the local average shear stiffness of the material close to the broken fibre, leading to a slightly faster stress recovery [3].

Fig. $5 \mathrm{~b}$ demonstrates the crucial difference between regular and random fibre packings. While any relative distance from the broken fibre is possible for random packings, regular packings only have data points at certain distances. Fibres in random packings can be much closer to the broken fibre, which can lead to SCFs almost twice as high as 
in regular packings. Data points for regular packings coincide reasonably well with the trend line for random packings.
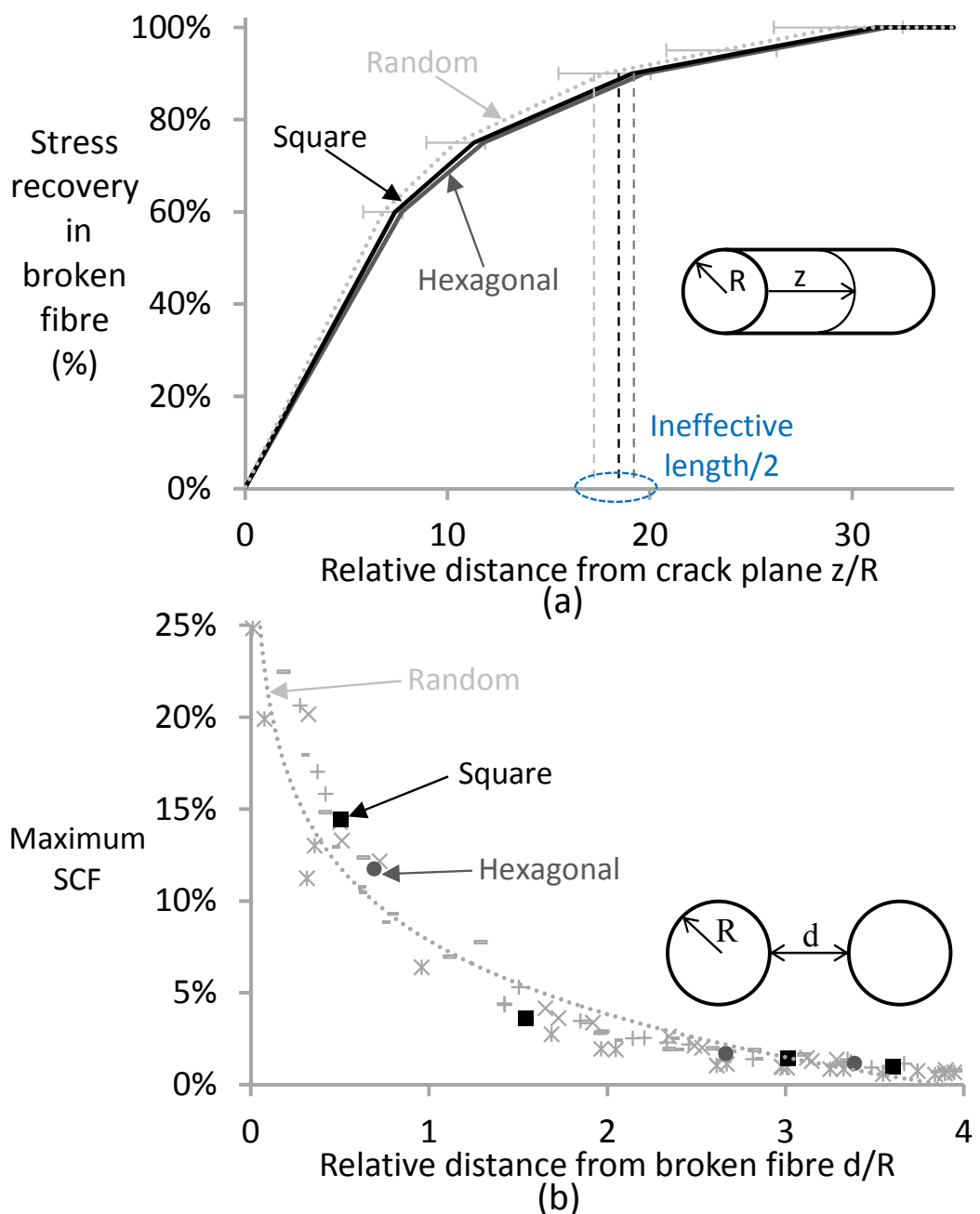

Figure 5: Stress redistributions for random, square and hexagonal packings: (a) piecewise linear interpolation of the stress recovery in the broken fibre with indication of the standard deviation for random fibre packings, and (b) stress concentration factors in the intact fibres. For the maximum SCF, the results of 5 simulations are shown with different light grey markers. The logarithmic trend line is entered into the strength model for random packings.

The stress redistributions were entered into the strength model and 50 simulations were performed for each packing type. Fig. 6 summarises the results for predicted failure strain. All observed differences are statistically significant, but are small nonetheless. The largest strain difference is only $0.06 \%$, which is smaller than the scatter caused by errors in the Weibull distribution. 


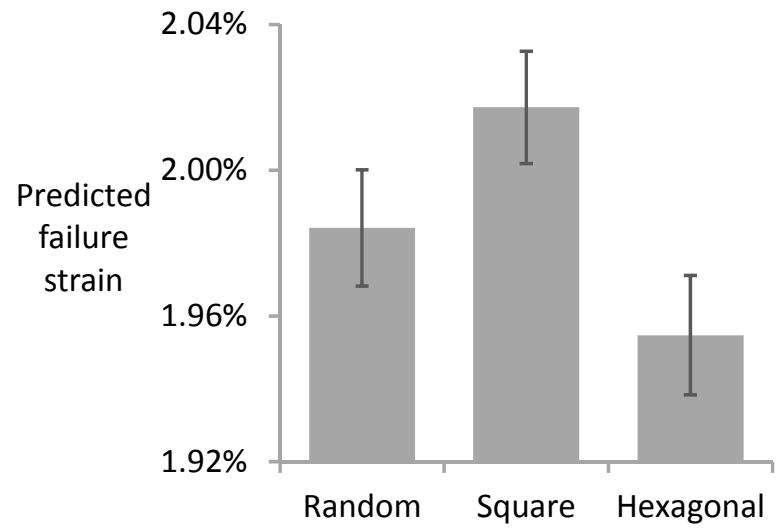

Figure 6: Predicted composites failure strains for models with the three packing types.

Fig. 7 proves that all packings show a similar cluster development up to a certain strain. Near final failure, however, the largest cluster is significantly larger for square packings. Fig. 6 and Fig. 7 indicate that the packing type has a minor influence on the failure strain and development. The fundamental reason can be found in Fig. 5b. The highest SCFs in random fibre packings are more than twice as high as in hexagonal packings. This does not mean that all SCFs are twice as high. Consider the 6 nearest neighbour fibres, which are all at the same distance for hexagonal packings, but at varying distances for random packings. In a specific random packing, for example the data points with a "+" marker in Fig. 5b, 3 fibres are close to the broken fibre and will carry a larger SCF than in hexagonal packings. The other fibres, however, will carry a smaller SCF than in hexagonal packings. These two aspects roughly cancel out each other, leading to a similar failure development and failure strain for all packing types. Similar trends were observed for a Weibull modulus of 8 .

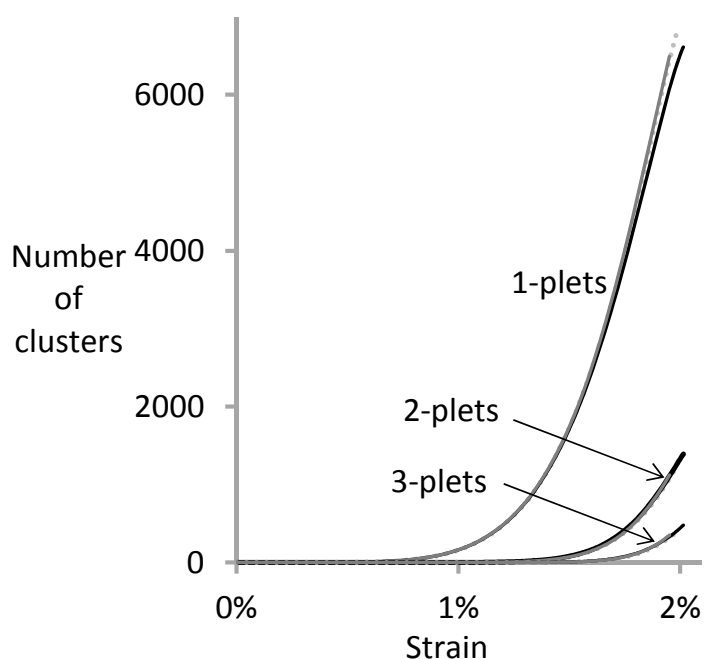

(a)

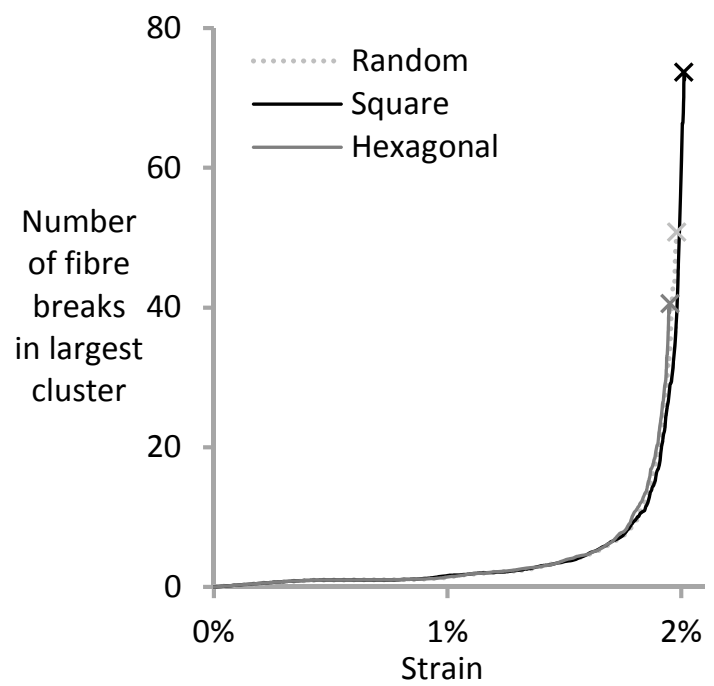

(b)

Figure 7: Cluster evolution for the three fibre packings: (a) 1-plet, 2-plet and 3-plet evolution, and (b) largest cluster evolution.

This is the first confirmation that square and hexagonal packings are accurate assumptions for modelling the $0^{\circ}$ tensile strength of UD composites. This conclusion is valid within the modelling assumptions presented earlier, but needs to be verified when other effects are taken into account. Including matrix plasticity or fibre-matrix debonding might lead to differences between predictions for different fibre packings. 
Random fibre packings can still be required in two cases: (1) for off-axis loading cases, and (2) in hybrid composites. The importance of random packings in off-axis loading has already been proven in literature [42]. In hybrid composites, the two fibre types often have different radiuses. Firstly, this difference would limit the maximum fibre volume fraction in regular, hybrid packings. Secondly, the dispersion of both fibre types would not be completely random, as their fibre centres would have fixed locations. This degree of dispersion is crucial in hybrid composites.

\subsection{Boundary effects}

The number of fibres in strength models is typically in the order of 1000 fibres. Typical tensile samples do not only contain more than a million fibres, but they are also longer than typical lengths used in strength models. Due to the stochastic variability of fibre strength, composite strength depends on the size of the tested samples $[33,43]$. Modelling results hence need to be interpreted with care. Apart from these size scaling effects, boundary effects can also be important in small RVEs. If a fibre breaks near the model perimeter, then that fibre will have fewer neighbouring fibres. Those neighbouring fibres will carry a larger SCF than if the break would occur in the centre of the model. This increases the probability for cluster formation near the perimeter.

Three approaches exist to deal with this problem. The first approach uses periodic boundary conditions at the edge of a model, typically applied to square RVEs $[31,44]$. A fibre break on one edge of the model would cause SCFs in fibres on the opposite edge. Implementing these boundary conditions in the presented model is challenging due to the random fibre packings, but not impossible. The second approach, which was used in this study, is more straightforward to implement in random fibre packings. In this approach, boundary fibres are added so that they can carry SCFs but are not allowed to break. Another approach is to neglect this issue altogether. While this may seem crude at first, this preferential cluster formation at the perimeter also occurs in real tests. In real tests however, the number of fibres is much larger than in models, thereby reducing the importance of fibres at the perimeter.

The role of boundary effects can be analysed by comparing models with and without unbreakable boundary fibres for different numbers of breakable fibres. For models with boundary fibres, a ring of boundary fibres is added that is about 2 fibres thick. The number of breakable fibres is the same as in the models without boundary fibres. This is necessary to avoid size scaling effects when comparing models with and without boundary fibres. Models with 250 breakable fibres contain 90 boundary fibres, while this number increases to 540 boundary fibres for models with 10000 breakable fibres. The relative number of boundary fibres hence decreases with increased model size.

Fig. 8 presents the predicted composite failure strains with and without boundary fibres. The failure strain of models with boundary fibres decreases with increased number of breakable fibres. This size scaling effect has been extensively described in literature $[33,43,45]$. Interestingly, such an effect is not observed in models without boundary fibres. In that case, cluster formation occurs preferentially near the perimeter and this become less likely for larger composites. This reduced probability will tend to increase the failure strain, and hence cancels out the size scaling effect. For small bundles, the boundary effect dominates the size scaling effect. Both model types converge with an increased number of breakable fibres. This convergence is expected, as the importance of the perimeter decreases with increased number of breakable fibres. 
DOI: 10.1016/j.compscitech.2015.04.002

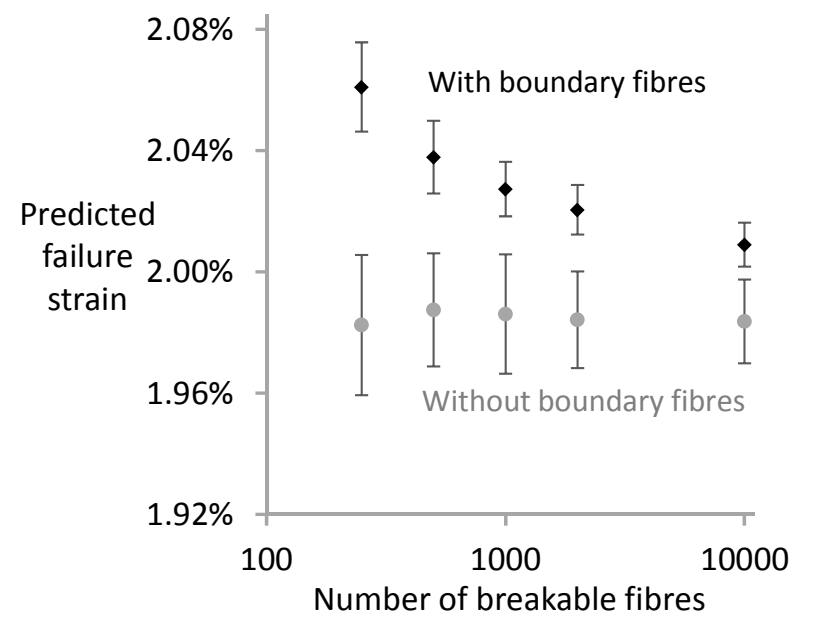

Figure 8: Predicted failure strain with and without boundary fibres as a function of the number of breakable fibres. All models are $10 \mathrm{~mm}$ long.

To further understand the origin of the difference in failure strain, the cluster development is analysed. The development of 1,2 and 3-plets was not affected by the boundary fibres, except near final failure (see Fig. 9a). The same conclusion was confirmed for larger clusters and models with a different number of breakable fibres.

The presence of boundary fibres does create larger critical cluster sizes (see Fig. 9b). Careful analysis of the locations of these critical clusters leads to a crucial difference between models with and without boundary fibres. Fig. 10 illustrates this difference by plotting a $2 \mathrm{D}$ view of all fibre breaks in the last strain increment before final failure. The colour of each fibre indicates the largest cluster along its length, thus providing a projection of the largest clusters in a $2 \mathrm{D}$ view. In models without boundary fibres, the critical cluster occurs near the model perimeter. In models with boundary fibres, however, the critical cluster occurs at random locations in the model. It is hypothesised that a smaller cluster is sufficient to cause unstable propagation in the absence of boundary fibres. This attributed to the fact that these clusters can develop near the perimeter, where they have fewer neighbours and hence higher SCFs. These higher SCFs will more rapidly lead to unstable propagation.

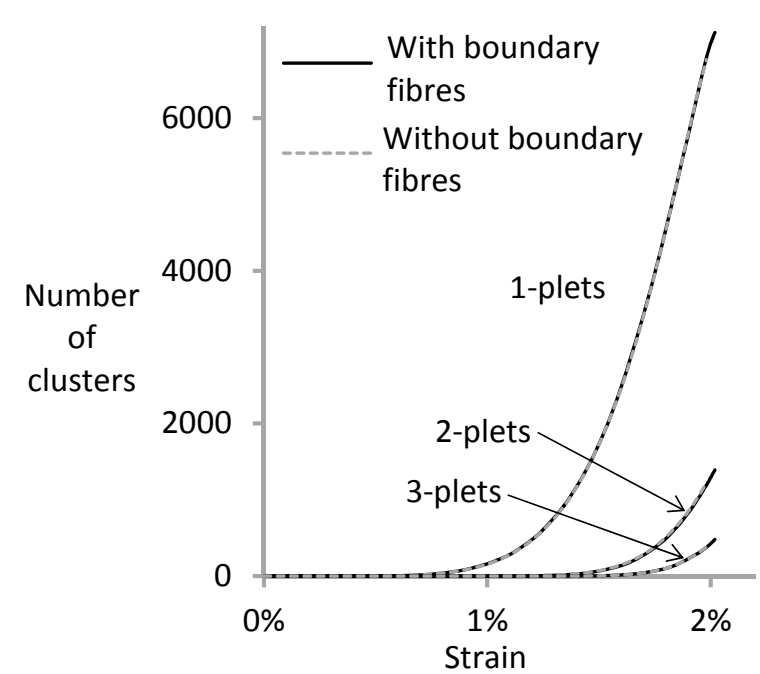

(a)

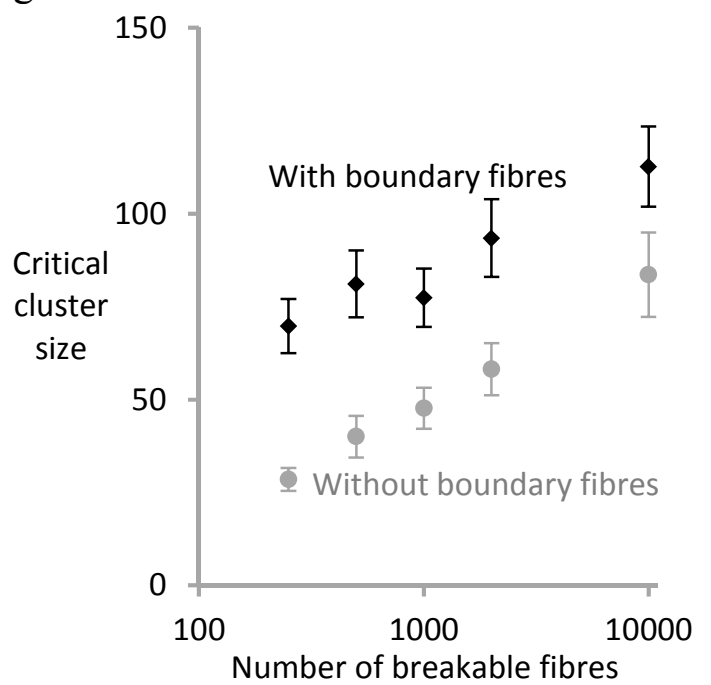

(b)

Figure 9: Failure development in models with and without boundary fibres: (a) the development of 1-plets, 2-plets and 3-plets for a model with 2000 breakable fibres, and (b) the critical cluster size as a function of the number of breakable fibres. 
Fig. $9 \mathrm{~b}$ also shows an increase in the critical cluster size with increased number of breakable fibres in the model. This increase is obvious for models without boundary fibres, but is less pronounced for models with boundary fibres. This agrees with the analytical equation for critical cluster size derived by Ibnabdeljalil and Curtin [44]. This equation was derived using periodic conditions at the perimeter, which is similar to models with boundary fibres. Their equation only depends on the Weibull modulus, and not on the number of fibres.

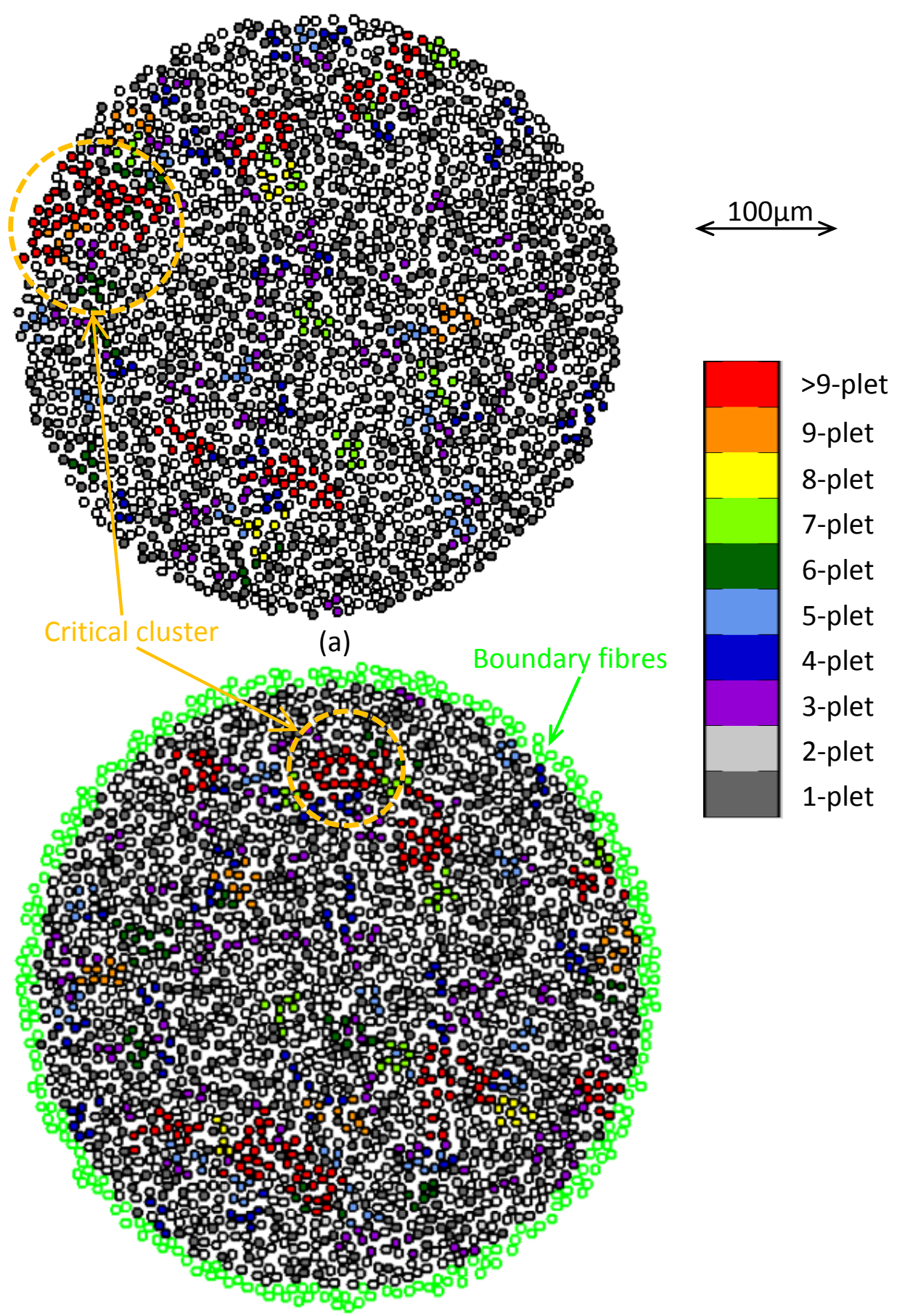

(b)

Figure 10: 2D view of the cluster development in models with 2000 breakable fibres: (a) without boundary fibres, and (b) with boundary fibres. Hollow black circles indicate that there are no fibre breaks along the length of the fibre, while hollow green circles represent the boundary fibres. The size of the largest cluster along the fibre is indicated by filling the circle with the corresponding color. The critical cluster is indicated by the large orange circle. 
The critical cluster size for both model types should converge for large number of fibres. Fig. 9b indicates a slow convergence, which is not yet reached for 10000 fibres.

It is difficult to decide whether boundary fibres should be included in strength models. The answer depends on the aim of the model. Without boundary fibres, the model corresponds to the experimental situation if the number of fibres is similar. If it is not computationally feasible to model the large number of fibres in the real samples, then boundary fibres make the model more representative of larger sample sizes.

\section{Conclusion}

Three potential issues in strength models for UD composites were assessed, leading to the following conclusions:

1. Determining a Weibull distribution based on 25-100 single fibre tests is insufficient to accurately predict the composite failure strain. Since single fibre tests are highly time-consuming, alternative methods to accurately determine Weibull distributions may need to be developed. Ideally, these methods would measure directly at the small gauge lengths that are relevant for strength models.

2. Even though stress redistributions after a fibre break are different in composites with random, square and hexagonal fibre packings, the differences in the predicted composite failure strain are negligible. This is the first confirmation that random and regular fibre packings have a similar failure strain and cluster development in a composite up to the final stage of its failure.

3. Boundary effects do not have a significant influence on the cluster development, except for near final failure. Boundary fibres can make the model more representative of larger samples, and may hence reduce simulation time.

Future developments in strength models for UD composites should focus on improved methods to determine Weibull distributions. X-ray computed tomography or single fibre fragmentation tests may be useful techniques to gather many data points in a short time.

\section{Acknowledgments}

The work leading to this publication has received funding from the European Union Seventh Framework Programme (FP7/2007-2013) under the topic NMP-2009-2.5-1, as part of the project HIVOCOMP (Grant Agreement No. 246389). The authors thank the Agency for Innovation by Science and Technology in Flanders (IWT) for the grant of Y. Swolfs. The authors also thank A.R. Melro and P. Camanho for the permission to use their random fibre packing generator. I. Verpoest holds the Toray Chair in Composite Materials at KU Leuven.

\section{References}

[1] Kaddour AS, Hinton MJ. Maturity of 3D failure criteria for fibre-reinforced composites: Comparison between theories and experiments: Part B of WWFE-II. Journal of Composite Materials. 2013;47(6-7):925-966.

[2] Christensen RM. The World Wide Failure Exercise II Examination of Results. Journal of Reinforced Plastics and Composites. 2013;32(21):1668-1672. 
[3] Swolfs Y, Gorbatikh L, Romanov V, Orlova S, Lomov SV, Verpoest I. Stress concentrations in an impregnated fibre bundle with random fibre packing. Composites Science and Technology. 2013;74:113-120.

[4] Hedgepeth JM. Stress concentrations in filamentary structures. NASA TN. 1961;D882( ):1-36.

[5] Phoenix SL, Schwartz P, Robinson HH. Statistics for the strength and lifetime in creep-rupture of model carbon/epoxy composites. Composites Science and Technology. 1988;32(2):81-120.

[6] Zweben C, Rosen BW. A statistical theory of material strength with application to composite materials. Journal of the Mechanics and Physics of Solids. 1970;18(3):189206.

[7] Smith RL, Phoenix SL, Greenfield MR, Henstenburg RB, Pitt RE. Lower-tail approximations for the probability of failure of three-dimensional fibrous composites with hexagonal geometry Proceedings of the Royal Society of London Series A: Mathematical Physical and Engineering Sciences. 1983;388(1795):353-391.

[8] Harlow DG, Phoenix SL. Probability distributions for the strength of composite materials II: A convergent sequence of tight bounds. International Journal of Fracture. 1981;17(6):601-630.

[9] Scop PM, Argon AS. Statistical theory of strength of laminated composites II. Journal of Composite Materials. 1969;3(JAN):30-47.

[10] Scott AE, Mavrogordato M, Wright P, Sinclair I, Spearing SM. In situ fibre fracture measurement in carbon-epoxy laminates using high resolution computed tomography. Composites Science and Technology. 2011;71:1471-1477.

[11] Scott AE, Sinclair I, Spearing SM, Thionnet A, Bunsell AR. Damage accumulation in a carbon/epoxy composite: Comparison between a multiscale model and computed tomography experimental results. Composites Part A: Applied Science and Manufacturing. 2012;43(9):1514-1522.

[12] Thionnet A, Chou HY, Bunsell A. Fibre break processes in unidirectional composites. Composites Part A: Applied Science and Manufacturing. 2014;65:148-160.

[13] Noda J, Nakada M, Miyano Y. Temperature dependence of accumulation of fiber breakages under tensile loading for unidirectional CFRP laminates. Journal of Reinforced Plastics and Composites. 2008;27(10):1005-1019.

[14] Swolfs Y, Gorbatikh L, Verpoest I. Fibre hybridisation in polymer composites: a review. Composites Part A: Applied Science and Manufacturing. 2014;67:181-200.

[15] Weibull W. A statistical distribution function of wide applicability. Journal of Applied Mechanics - Transactions of the ASME. 1951;18(3):293-297.

[16] Curtin WA, Takeda N. Tensile strength of fiber-reinforced composites: II. Application to polymer matrix composites. Journal of Composite Materials. 1998;32(22):2060-2081.

[17] Rosen BW. Tensile failure of fibrous composites. AIAA Journal. 1964;2(11):19851991.

[18] Gutans J, Tamuzs V. Strength probability of unidirectional hybrid composites. Theoretical and Applied Fracture Mechanics. 1987;7(3):193-200.

[19] Gulino R, Phoenix SL. Weibull strength statistics for graphite fibres measured from the break progression in a model graphite/glass/epoxy composite. Journal of Materials Science. 1991;26(11):3107-3118.

[20] Beyerlein IJ, Phoenix SL. Statistics for the strength and size effects of microcomposites with four carbon fibers in epoxy resin. Composites Science and Technology. 1996;56(1):75-92.

[21] Watson AS, Smith RL. An examination of statistical theories for fibrous materials in the light of experimental data. Journal of Materials Science. 1985;20(9):3260-3270. 
[22] Thomason JL. On the application of Weibull analysis to experimentally determined single fibre strength distributions. Composites Science and Technology. 2013;77:74-80.

[23] Zinck P, Mader E, Gerard JF. Role of silane coupling agent and polymeric film former for tailoring glass fiber sizings from tensile strength measurements. Journal of Materials Science. 2001;36(21):5245-5252.

[24] Naito K, Yang JM, Tanaka Y, Kagawa Y. The effect of gauge length on tensile strength and Weibull modulus of polyacrylonitrile (PAN)- and pitch-based carbon fibers. Journal of Materials Science. 2012;47(2):632-642.

[25] Otani H, Phoenix SL, Petrina P. Matrix effects on lifetime statistics for carbon fiber/epoxy microcomposites in creep-rupture. Journal of Materials Science. 1991;26(7):1955-1970.

[26] Watanabe J, Tanaka F, Okabe T. The tensile strength distribution of carbon fibers at short gauge length. 38th Conference of the Japan Society for Composite Materials, 2013. p. 171-172.

[27] Berger $\mathrm{MH}$, Jeulin D. Statistical analysis of the failure stresses of ceramic fibres: Dependence of the Weibull parameters on the gauge length, diameter variation and fluctuation of defect density. Journal of Materials Science. 2003;38(13):2913-2923.

[28] Johnson AC, Zhao FM, Hayes SA, Jones FR. Influence of a matrix crack on stress transfer to an alpha-alumina fibre in epoxy resin using FEA and photoelasticity. Composites Science and Technology. 2006;66(13):2023-2029.

[29] Beyerlein IJ, Phoenix SL. Stress concentrations around multiple fiber breaks in an elastic matrix with local yielding or debonding using quadratic influence superposition. Journal of the Mechanics and Physics of Solids. 1996;44(12):1997-2039.

[30] Beyerlein IJ, Phoenix SL, Sastry AM. Comparison of shear-lag theory and continuum fracture mechanics for modeling fiber and matrix stresses in an elastic cracked composite lamina. International Journal of Solids and Structures. 1996;33(18):2543-2574.

[31] Okabe T, Sekine H, Ishii K, Nishikawa M, Takeda N. Numerical method for failure simulation of unidirectional fiber-reinforced composites with spring element model. Composites Science and Technology. 2005;65(6):921-933.

[32] Swolfs Y, McMeeking RM, Verpoest I, Gorbatikh L. Matrix cracks around fibre breaks and their effect on stress redistribution and failure development in unidirectional composites. Composites Science and Technology. 2015;108:16-22.

[33] Pimenta S, Pinho ST. Hierarchical scaling law for the strength of composite fibre bundles. Journal of the Mechanics and Physics of Solids. 2013;61(6):1337-1356.

[34] Blassiau S, Thionnet A, Bunsell AR. Micromechanisms of load transfer in a unidirectional carbon fibre-reinforced epoxy composite due to fibre failures. Part 1: Micromechanisms and 3D analysis of load transfer: The elastic case. Composite Structures. 2006;74(3):303-318.

[35] Xia Z, Curtin WA, Peters PWM. Multiscale modeling of failure in metal matrix composites. Acta Materialia. 2001;49(2):273-287.

[36] Nedele MR, Wisnom MR. Three-dimensional finite element analysis of the stress concentration at a single fibre break. Composites Science and Technology. 1994;51(4):517-524.

[37] Romanov V, Lomov SV, Swolfs Y, Orlova S, Gorbatikh L, Verpoest I. Statistical analysis of real and simulated fibre arrangements in unidirectional composites. Composites Science and Technology. 2013;87:126-134.

[38] Smith RL. The random variation of stress concentration factors in fibrous composites. Journal of Materials Science Letters. 1983;2(8):385-387.

[39] Swolfs Y, Gorbatikh L, Verpoest I. Stress concentrations in hybrid unidirectional fibre-reinforced composites with random fibre packings. Composites Science and Technology. 2013;85:10-16. 
[40] Swolfs Y, McMeeking RM, Gorbatikh L, Verpoest I. The effect of fibre dispersion on initial failure strain and cluster development in unidirectional carbon/glass hybrid composites. Composites Part A: Applied Science and Manufacturing. 2015;69:279-287. [41] Phoenix SL, Sexsmith RG. Clamp effects in fiber testing. Journal of Composite Materials. 1972;6(JUL):322-337.

[42] Trias D, Costa J, Mayugo JA, Hurtado JE. Random models versus periodic models for fibre reinforced composites. Computational Materials Science. 2006;38(2):316-324.

[43] Curtin WA. Size scaling of strength in heterogeneous materials. Physical Review Letters. 1998;80(7):1445-1448.

[44] Ibnabdeljalil M, Curtin WA. Strength and reliability of fiber-reinforced composites: Localized load-sharing and associated size effects. International Journal of Solids and Structures. 1997;34(21):2649-2668.

[45] Wisnom MR, Khan B, Hallett SR. Size effects in unnotched tensile strength of unidirectional and quasi-isotropic carbon/epoxy composites. Composite Structures. 2008;84(1):21-28. 